\title{
Traversable wormholes: the Roman ring
}

\author{
Matt Vissert \\ Physics Department, Washington University, St. Louis, Missouri 63130-4899 \\ (gr-qc/9702043; 11 November 1996)
}

In this brief report I introduce a yet another class of geometries for which semi-classical chronology protection theorems are of dubious physical reliability. I consider a "Roman ring" of traversable wormholes, wherein a number of wormholes are arranged in a ring in such a manner that no subset of wormholes is near to chronology violation, though the combined system can be arbitrarily close to chronology violation. I show that (with enough wormholes in the ring) the gravitational vacuum polarization (the expectation value of the quantum stress-energy tensor) can be made arbitrarily small. In particular the back-reaction can be kept arbitrarily small all the way to the "reliability horizon" - so that semi-classical quantum gravity becomes unreliable before the gravitational back reaction becomes large.

Accepted for publication in Physical Review D15. 


\section{INTRODUCTION}

Working within the context of semi-classical quantum gravity, Krasnikov [1] and Sushkov [2] have recently provided examples of two classes of spacetimes containing time machines for which the gravitational vacuum polarization is arbitrarily small all the way to the chronology horizon. In related developments Kay, Radzikowski, and Wald [3], and Cramer and Kay [4] have shown that these geometries suffer from diseases on the chronology horizon itself. More recently, I have argued [5] that we should not physically trust semi-classical quantum gravity once we reach the chronology horizon.

In this brief report I wish to present yet another class of spacetimes for which the gravitational vacuum polarization can be made arbitrarily small. Implications for chronology protection [6,7] are briefly discussed.

\section{THE ROMAN RING}

Given one wormhole, it appears (classically) to be absurdly easy to turn it into a time machine [8 11], though quantum effects vitiate this particular approach [6, 7, 10, 12].

Given two wormholes, it appears (even including quantum effects) to be relatively easy to turn the compound system into a time machine without each individual wormhole itself being a time machine 13, 14.

Given many wormholes, I shall now show that it appears to be even easier to turn the conglomeration into a time machine (with all sub-collections of wormholes not themselves being time machines).

The key technical result is that, for any spacetime of non-trivial topology, the gravitational vacuum polarization may be estimated by adiabatic techniques to be

$$
\left\langle T^{\mu \nu}(x)\right\rangle \approx \Delta_{\gamma} \frac{\hbar}{s_{\gamma}(x, x)^{4}} t^{\mu \nu} .
$$

Here $s_{\gamma}(x, x)$ is the length of the shortest spacelike geodesic connecting the point $x$ to itself, while $t^{\mu \nu}$ is a dimensionless tensor built out of the metric and tangent vectors to this geodesic. $\Delta_{\gamma}$ is the van Vleck determinant associated with this geodesic.

For the one-wormhole system this van Vleck determinant can be estimated (insofar as the throat of the wormhole is reasonably thin) to be close to 1 . For the twowormhole system the van Vleck determinant is a complicated function of relative positions and velocities. I will now provide a simple class of multiple wormhole configurations in which the van Vleck determinant is calculable - and thereby show that there exists a class of geometries for which the van Vleck determinant can be made arbitrarily small all the way down to the chronology horizon.

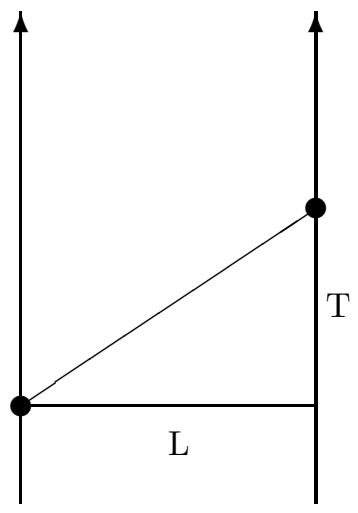

FIG. 1. Schematic representation of a single (chronology respecting) wormhole. We identify two timelike lines that are separated by a spatial jump $L$ and time shift $T$, with $T \ll L$.

Start by taking $N$ identical wormholes in otherwise flat Minkowski spacetime. (These may be taken to be simple cut-and-paste wormholes of the type discussed in 12, 16, 17. .) For simplicity assume that all wormhole mouths are at rest with respect to each other; so each wormhole is characterized by a spatial jump $L$ and timeshift $T$ with $T \ll L$.

If we look at a geodesic that wraps once through a single wormhole, the invariant interval is simply $s_{1}^{2}=$ $L^{2}-T^{2} \gg 0$.

Now arrange the $N$ wormholes in a big symmetric polygon, so that the exit mouth of one wormhole is a normalspace distance of $\ell$ from the entrance mouth of the next wormhole. (We will want $\ell \ll L$, so that the normal space distance travelled to get from one wormhole to the next is less than the distance then "jumped" by going through the wormhole.)

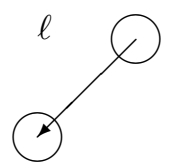

$\mathrm{L}$

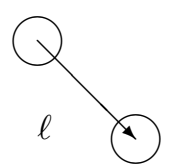

$\mathrm{L}$

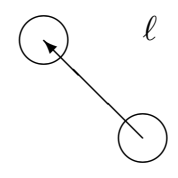

$\mathrm{L}$

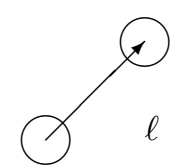

FIG. 2. Schematic representation of a Roman ring. This example contains four wormholes. In each wormhole the two mouths are separated by a spatial jump $L$. The normal space distance from the exit mouth of one wormhole to the entrance mouth of the next is $\ell$.

This "Roman ring" is a generalization of the twowormhole "Roman configuration" 8, 12 14]. (The realization that wormholes generically seem to imply time 
travel can be traced back to an observation by Tom Roman 11]. The "Roman configuration" was the first two-wormhole time machine constructed by Morris and Thorne [8].) The $N$-wormhole "Roman ring" can holistically be close to forming a time machine even if its individual components are perfectly well behaved.

Consider a closed geodesic that wraps once around the entire compound system: this consists of $N$ segments of length $\ell$ in normal space, plus $N$ time-shifts of magnitude $T$ from going through the $N$ wormholes. The invariant interval of this once-through-the-system geodesic is

$$
s_{\gamma}^{2}=(N \ell)^{2}-(N T)^{2}=N^{2}\left(\ell^{2}-T^{2}\right) .
$$

Because it is the normal-space distance between the wormhole mouths that enters here $(\ell$; not the spatial jump $L$ ) we can easily make this geodesic timelike.

For a conformally coupled massless field the symmetries enforce

$$
t^{\mu \nu}=\eta^{\mu \nu}-4 t^{\mu} t^{\nu}
$$

Here $\eta^{\mu \nu}$ is the spacetime metric, which is flat except at the wormholes themselves; $t^{\mu}$ is the tangent vector to the geodesic and is given (up to rotations) by

$$
t^{\mu}=\frac{(T, 0,0, \ell)}{\sqrt{\ell^{2}-T^{2}}}=\frac{N(T, 0,0, \ell)}{s_{\gamma}} .
$$

So anywhere along the geodesic $\gamma$ we can estimate

$$
\left\langle T^{\mu \nu}\right\rangle \approx \Delta_{\gamma} \frac{\hbar}{s_{\gamma}^{4}}\left[\eta^{\mu \nu}-4 \frac{N^{2}(T, 0,0, \ell)^{\mu}(T, 0,0, \ell)^{\nu}}{s_{\gamma}^{2}}\right] .
$$

Of course, the only reason we are bothering with this Roman ring geometry is because the extreme symmetry makes it relatively easy to calculate the van Vleck determinant. Using the thin-throat approximation, plus the tidal reformulation of the van Vleck evolution equation presented in 15], we may calculate the van Vleck determinant at the surface of any of the wormhole throats to be 13

$$
\Delta_{\gamma}=\left[\frac{N}{U_{N-1}\left(1+\frac{\ell}{R}\right)}\right]^{2}
$$

Here $U_{N}(x)$ is a Chebyshev polynomial of the second kind, and $R$ is the radius of each wormhole mouth (assumed spherical). The time shift $T$, and spatial jump $L$ quietly cancel out of the calculation for the van Vleck determinant.

Proving the above result is a combinatoric agony that is presented in excruciating detail in [13]. That calculation was carried out for a slightly different configuration: a geodesic that wraps $N$ times through a single wormhole, but that calculation can just as easily be adapted to the present case; a geodesic that wraps once through $N$ wormholes - note that the high degree of symmetry in the Roman ring configuration is essential for this purpose.
If we are satisfied with the situation $\ell \gg R$ (a perfectly sensible constraint - manipulating wormholes is likely to be quite difficult enough without having them bump into each other) then we can approximate

$$
\Delta_{\gamma} \approx N^{2}\left(\frac{R}{2 \ell}\right)^{2(N-1)}
$$

As a consistency check, compare this with the results quoted by Kim and Thorne [10], Lyutikov [14, and Visser [13.

The physical interpretation for this result is simple: the van Vleck determinant measures geometrically induced deviations from the inverse-square law [12]. By assumption, we are sitting right on top of one wormhole mouth, and the above result can be thought of as due to $N-1$ defocussing events which occur as we move through the $N-1$ other wormholes in the system to get back to our starting point.

The van Vleck determinant may now be made as small as desired simply by adding more wormholes to the system.

At the throat of any one of the wormholes, for any $\ell>T$, we have

$$
\begin{aligned}
\left\langle T^{\mu \nu}\right\rangle \approx & \hbar \frac{N^{2}}{s_{\gamma}^{4}}\left(\frac{R}{2 \ell}\right)^{2(N-1)} \\
& \times\left[\eta^{\mu \nu}-4 N^{2} \frac{(T, 0,0, \ell)^{\mu}(T, 0,0, \ell)^{\nu}}{s_{\gamma}^{2}}\right] .
\end{aligned}
$$

So at fixed $T$ and $\ell$ we have

$$
\begin{aligned}
\left\langle T^{\mu \nu}\right\rangle \approx & \hbar \frac{1}{N^{2}\left(\ell^{2}-T^{2}\right)^{2}}\left(\frac{R}{2 \ell}\right)^{2(N-1)} \\
& \times\left[\eta^{\mu \nu}-4 \frac{(T, 0,0, \ell)^{\mu}(T, 0,0, \ell)^{\nu}}{\ell^{2}-T^{2}}\right] .
\end{aligned}
$$

In particular $\left\langle T^{\mu \nu}\right\rangle \rightarrow 0$ as $N \rightarrow \infty$.

Now suppose the whole system is adiabatically shrunk, keeping $T$ fixed but letting $\ell \rightarrow T^{+}$. The "reliability horizon" [5], the location at which we should cease to believe the applicability of semi-classical quantum gravity, will be located at $\sqrt{\ell^{2}-T^{2}}=\ell_{\text {Planck }}$.

(General arguments supporting this designation are provided in [5]. In the present more specific context it suffices to realise that once $\sqrt{\ell^{2}-T^{2}}<\ell_{\text {Planck }}$, any quantum field [including gravitons] propagating on this background will be subject to Planck scale physics.)

At the throat of any one wormhole, when the system is at the reliability horizon, we have

$$
\begin{aligned}
\left\langle T^{\mu \nu}\right\rangle \approx & \hbar \frac{1}{N^{2} \ell_{\text {Planck }}^{4}}\left(\frac{R}{2 \ell}\right)^{2(N-1)} \\
& \times\left[\eta^{\mu \nu}-4 \frac{(\ell, 0,0, \ell)^{\mu}(\ell, 0,0, \ell)^{\nu}}{\ell_{\text {Planck }}^{2}}\right] .
\end{aligned}
$$

Again $\left\langle T^{\mu \nu}\right\rangle \rightarrow 0$ as $N \rightarrow \infty$. 
Thus with enough wormholes, we can arrange the gravitational vacuum polarization, and therefore the backreaction, to be arbitrarily small all the way down to the reliability horizon.

\section{IMPLICATIONS}

This counter-example is enough to show that it is impossible to come up with a chronology protection theorem that makes reference only to the "reliable region"and so it is impossible to come up with chronology protection theorem that is physically reliable within the context of semi-classical quantum gravity.

In this regard I am completely in agreement with Krasnikov [1] and Sushkov [2], though the current class of models is obtained in a radically different (and perhaps more physically transparent) manner.

My interpretation is perhaps a little different: I view this not as a vindication for time travel enthusiasts but rather as an indication that resolving issues of chronology protection requires a fully developed theory of quantum gravity [5].

\section{ACKNOWLEDGMENTS}

This research was supported by the U.S. Department of Energy. I wish to thank Tom Roman for reading the manuscript and providing helpful comments.

* $\quad$ Electronic mail: visser@kiwi.wustl.edu

[1] S. V. Krasnikov, On the quantum stability of the time machine, Phys. Rev. D54, 7322-7327, (1996); grqc/9508038.

[2] S. V. Sushkov, Chronology protection and quantized fields: Complex automorphic scalar field in Misner space, Class. Quant. Grav. 14, 523-534, (1997); gr-qc/9509056.

[3] B. S. Kay, M. Radzikowski, and R. M. Wald, Quantum Field Theory on Spacetimes with a Compactly Generated Cauchy Horizon, 1996, gr-qc/9603012.

[4] C. R. Cramer and B. S. Kay, Stress-energy must be singular on the Misner space horizon even for automorphic fields, Class. Quant. Grav. 13, L143-L149, (1996); grqc/9606027.

[5] M. Visser, A reliability horizon for semi-classical quantum gravity, 1997, gr-qc/9702041.

[6] S. W. Hawking, "The Chronology Protection Conjecture", in Proceedings of the 6th Marcel Grossmann Meeting, Kyoto, Japan, June 23-29, 1991, edited by H. Sato, (World Scientific, Singapore, 1992).

[7] S. W. Hawking, Phys. Rev. D46, 603 (1992).

[8] M. S. Morris and K. S. Thorne, Am. J. Phys. 56, 395 (1988).
[9] M. S. Morris, K. S. Thorne, and U. Yurtsever, Phys. Rev. Lett. 61, 1446 (1988).

[10] S. W. Kim and K. S. Thorne, Phys. Rev. D43, 3929 (1991).

[11] K. S. Thorne, Black holes and time warps-Einstein's outrageous legacy, (Norton, New York, 1994).

[12] M. Visser, Lorentzian wormholes-from Einstein to Hawking, (AIP Press, New York, 1995).

[13] M. Visser, Phys. Rev. D49, 3963 (1994); gr-qc/9311026.

[14] M. Lyutikov, Phys. Rev. D49, 4041 (1994).

[15] M. Visser, Phys. Rev. D47, 2395 (1993); hepth/9303020

[16] M. Visser, Phys. Rev. D39, 3182 (1989).

[17] M. Visser, Nucl. Phys. B328, 203 (1989). 